\title{
La diseminación de la arquitectura en la era digital. El ecosistema de las revistas (digitales), repositorios y redes sociales
} Dissemination of Architecture in the Digital Era. The Ecosystem of (Digital) Magazines, Repositories and Social Networks

\author{
Guido Cimadomo ${ }^{[1]}$, Rubén García Rubio ${ }^{[2]}$, Vishal Shahdadpuri Aswani ${ }^{[3]}$ \\ ${ }^{[1]}$ Universidad de Málaga, España, ${ }^{[2]}$ Tulane University, EE. UU. \\ ${ }^{[3]}$ eAM' tech lab, Universidad de Málaga, España \\ Traducción Translation Traducido por los autores
}

\section{Palabras clave Keywords}

Diseminación digital, publicaciones arquitectónicas, repositorios online, redes sociales, proyecto arquitectónico, Historia de la arquitectura, crítica arquitectónica, ArchDaily, FIG Projects

Digital dissemination, architectural publications, online repositories, social networks, architectural design, history of architecture, architectural criticism, ArchDaily, FIG Projects

\section{Resumen}

A partir de la transformación que la web 2.0 ofrece en el ámbito de la difusión de la producción arquitectónica a través del concepto de shared knowledge, se producen unas mutaciones en los criterios de valoración e impacto de su diseminación. Utilizando herramientas de analítica web, nos proponemos comprender el ecosistema digital de difusión de la arquitectura en la era digital. Se profundiza el estudio con una entrevista a FIG Projects, debido al impacto que tienen perfiles similares con estructuras menos empresariales comparados con los portales que dominan las estadísticas analizadas. Se reconoce así tanto el nuevo carácter de la difusión arquitectónica y la transformación de las reglas tradicionales, como el papel de las redes sociales en dicha diseminación, al convertir al usuario en productor, editor y crítico de arquitectura.
Abstract

From the transformation that web 2.0 offers in disseminating architectural production through the concept of shared knowledge, there are mutations in the evaluation criteria and the impact of its dissemination. Using web analytics tools, we aim to understand the digital ecosystem to disseminate architecture in the digital age. The study is deepened with an interview with FIG Projects due to the impact that similar profiles have with fewer business structures than the portals that dominate the statistics analyzed. Thus, both the new character of architectural dissemination and the transformation of traditional rules are recognized, as well as the role of social networks in such dissemination, by turning the user into a producer, editor and critic of architecture. 
"Internet, el correo electrónico, los blogs, Google, Twitter, YouTube, Facebook, etc., han cambiado profundamente la manera en que trabajamos, escribimos, analizamos, interactuamos, y jugamos. ¿Podemos esperar que la arquitectura no se vea afectada?". (1)

En el libro Privacidad y publicidad: la arquitectura moderna como medio de comunicación de masas, Beatriz Colomina analiza la relación entre la comunicación y la práctica arquitectónica, con una por aquel momento -el libro es la adaptación de su tesis doctoral de 1991-, rompedora pregunta sobre la validez de considerar la arquitectura misma como un medio de comunicación. En el prólogo a la edición española -publicado con dieciséis años de diferencia con respecto a la versión original en inglés-, la autora vuelve a señalar la existencia de una 'revolución' en la sociedad actual similar a la que experimentaron los arquitectos de principios del siglo pasado. (Fig. 1)

Los portales de arquitectura monotemáticos surgidos con la web 2.0 ofrecen todos los días un número relevante de contenidos arquitectónicos, hasta el punto de estar creando un verdadero repositorio de la producción arquitectónica a escala global. (Fig. 2) Archdaily, Dezeen, Designboom, Architizer, Archinect, o Divisare, por citar algunos de los más conocidos y con mayor impacto, han reemplazado a otros medios tradicionales de diseminación, en la difusión de noticias y proyectos relacionados con la arquitectura. Por ejemplo, Archdaily publica una media de 20 posts diarios-incluyendo obra arquitectónica, noticias o artículos de miscelánea-, frente a los 2-3 elementos que comparte el portal Arquitectura Viva.

La democratización de la difusión del proyecto arquitectónico, que hoy en día permite a los mismos autores difundir a escala global su trabajo, hace irrelevantes las limitaciones de transmisión del medio físico. Este modelo tiene, sin embargo, otros aspectos menos positivos que es necesario destacar. Por un lado, se evidencia la dicotomía entre 'el objeto para ser visto' y el 'objeto para funcionar', utilizando los términos empleados hace ya 50 años por Oriol Bohigas. (3) Hoy en día el proyecto, y más frecuentemente su

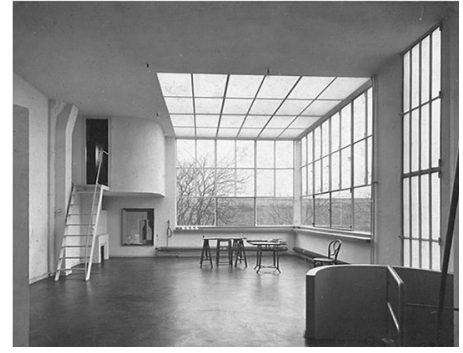

Fig. 1. Fotografía de la Maison Ozenfant, 1922. Le Corbusier. La edición por parte del autor incluía una especial atención a la composición troncocónica, a la utilización de un falso punto de fuga y a la representación antropomórfica como estrategias para una arquitectura de lo posible. (2)

"The internet, email, blogs, Google, Twitter, YouTube, Facebook, etc., have profoundly changed how we work, write, analyze, interact, and play. Can we expect that architecture is not affected?". (1)

In the book Privacy and Publicity: Modern Architecture as Mass Media, Beatriz Colomina analyses the relationship between communication and architectural practice, with a groundbreaking question at the time -the book is an adaptation of her 1991 doctoral thesis-, about the validity of considering architecture itself as a means of communication. In the prologue to the Spanish edition -published sixteen years later than the original English version-, the author again points out the existence of a 'revolution' in today's society similar to that experienced by architects at the beginning of the last century. (Fig. 1)

The monothematic architectural portals that emerged with web 2.0 offer a relevant number of architectural contents every day, to the point of creating a real repository of architectural production on a global scale. (Fig. 2) Archdaily, Dezeen, Designboom, Architizer, Archinect, or Divisare, to name a few of the best known and with the greatest impact, have replaced other traditional means of dissemination of news and projects related to architecture. For example, Archdaily publishes an average of 20 daily posts -including architectural work, news or miscellaneous articles-, compared to the 2-3 elements shared by the Arquitectura Viva portal/website.

The democratization of the dissemination of architectural design, which nowadays allows the same authors to disseminate their work on a global scale, makes the limitations of transmission of the physical medium irrelevant. This model has, however, other 

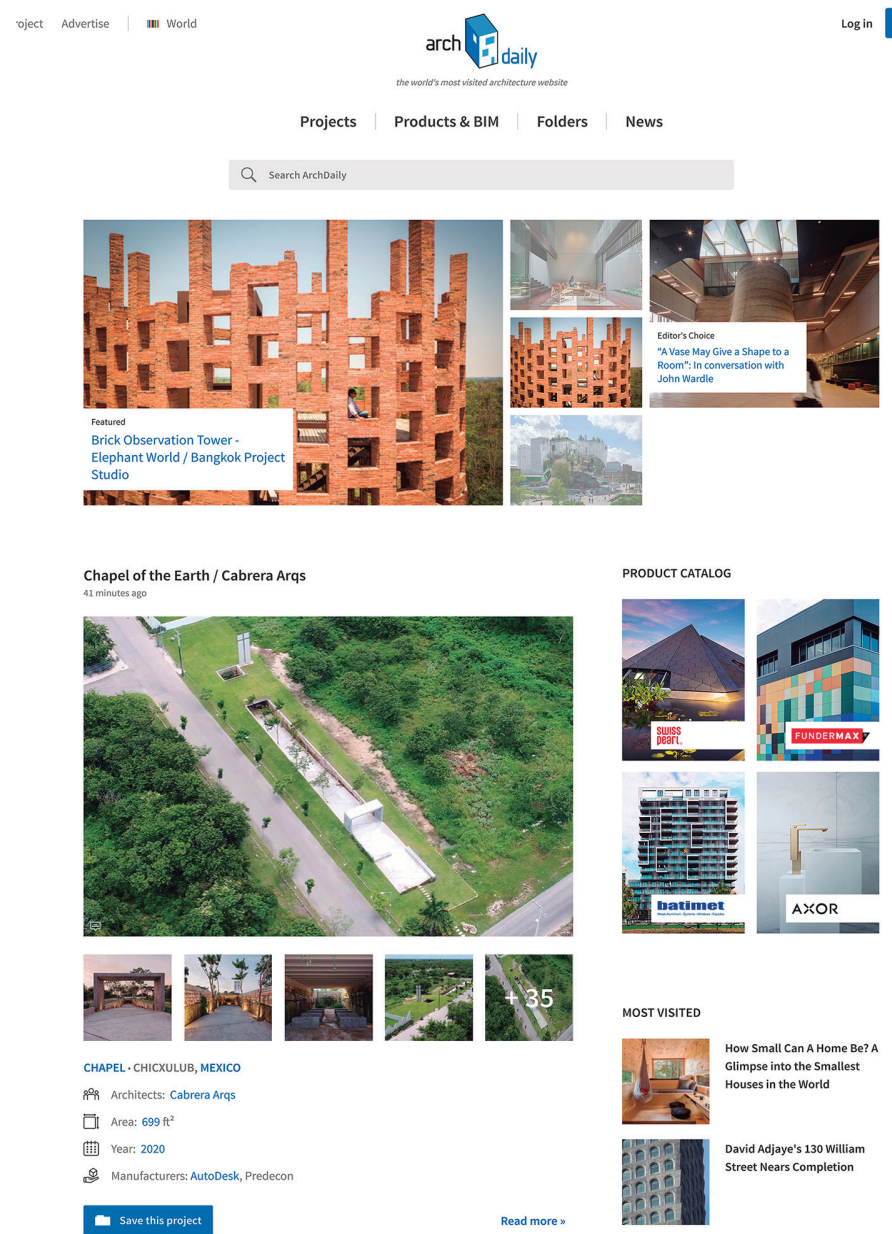

Fig. 2. Portada de la web del repositorio monotemático ArchDaily.com

less positive aspects that need to be highlighted. On the one hand, the dichotomy between 'the object to be seen' and 'the object to function' is evident, using the terms used by Oriol Bohigas fifty years ago. (3) Nowadays, the project, and more frequently its representation, is made to be seen, to be valued expressively and volumetrically, more than for its adaptation to the environment, to the programmatic, social or economic needs of its future users. (Fig. 3) In this same line, Jorge Sainz warned us at the beginning of the 90s of the greater importance of photography -and, by extension, the digital image- over the drawing, which has always been at the center of the architect's training. This reflection finds its definitive pragmatic adaptation in the current context. (4) This trend is currently perceived and sanctioned by Winy Maas, when stating that "Architecture is no longer the art of building [...] Architects seem to have exchanged real space for screens and glossy pages. Today, the art of architectural publishing -both print and online- is the key to building a prestigious empire; appearing in print -or in a viral blog- is what creates a star figure". (5)

The new forms of online architectural dissemination generate new criteria for its evaluation and impact. If previously quality was the main criterion, the digital world includes other types of strategies based on parameters and algorithms, not always easy to understand. For this reason, a question arises spontaneously about the direction being taken by the dissemination of the discipline and, especially, of its works and the effects on new generations of architects. Rafael Moneo already raised this reflection when recognizing the possibility of an increasing individuality of the architectural process, often detached from history. (6) Although the feedback produced by the images we are exposed to daily must also be recognized, it generates a process of evolution and adaptation concerning previous works according to the theory of the "variant inflects model" suggested by Dana Cuff. (7) 
representación, viene realizado para ser visto, para ser valorado expresiva y volumétricamente, más que por su adaptación al medio, a las necesidades programáticas, sociales o económicas de sus futuros usuarios. (Fig. 3) En esta misma línea, Jorge Sainz nos advertía a principios de los años 90 de la mayor importancia de la fotografía -y por extensión la imagen digitalsobre el dibujo, desde siempre al centro de la formación del arquitecto. Reflexión que encuentra en definitiva su adaptación pragmática en el contexto actual. (4) Esta tendencia viene percibida y sancionada en la actualidad por Winy Maas, al manifestar que "la arquitectura ya no es el arte de construir
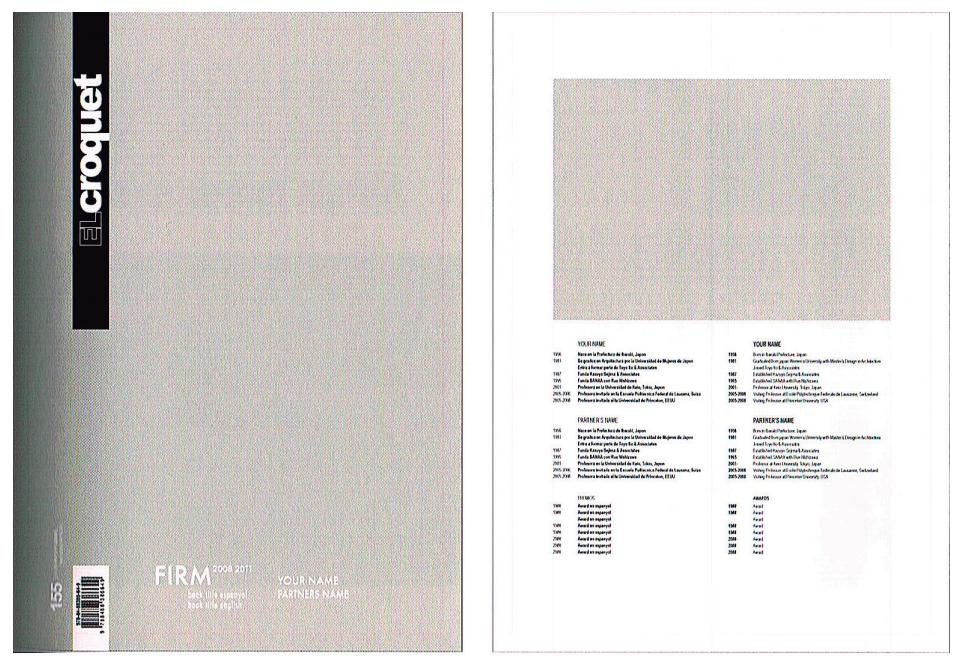

Portrait

Insert an image of yourself here (preferably a $3 / 4$-lenght portrait in elegant black clothes against a neutral background)
Fig. 3. Sátira crítica del esquema tradicional de una revista de $E l$ Croquis, que ofrece democratizar esta publicación exclusiva para cada arquitecto deseoso de fama y prestigio [ver 5].

This paper is part of a broader research on the transformation of architectural diffusion in digital media. It aims to understand the changes taking place in how the history of architecture is being told in the 21st Century and, in particular, the trends originated by the incessant and progressive use of the internet and its relationships with traditional media. This text mainly uses the typical tools of network metrics to expose the ecosystem of digital architectural diffusion today. Subsequently, the essay analyzes a specific profile active in Facebook, FIG Projects, to highlight the role of social networks in disseminating architecture and to evidence the role of the critic or editor of 'yesteryear' compared to other profiles of a larger scale and structure. Thus, both the new character of architectural dissemination and the role of social networks in it is recognized.

The Impact of Digital Media in the Field of Architecture. To understand the current trends in disseminating architecture, a comparative analysis is made between the main media dedicated to diffusing it. As a large part of today's dissemination is based on the digital sphere and, considering the permanent increase in users, (Figs. 4 and 5) the analysis focuses exclusively on the digital world.

The tool used is Alexa.com, one of the most recognized applications for measuring traffic, relevance and positioning of websites. The analysis has focused on both the most relevant digital architecture repositories and the online versions of the most authoritative traditional media. Specifically, the study has mainly evaluated three aspects offered by Alexa in its statistics: the daily visualizations per visitor, the time spent per visitor and, above all, the position in the global ranking based on "the traffic and global interaction of the web in the last 90 days" as the web itself explains. (Fig. 6) 
[...] Los arquitectos parecen haber cambiado el espacio real por pantallas y páginas brillantes. Actualmente, el arte de la publicación arquitectónica -tanto impresa como online- es la clave para construir un prestigioso imperio; aparecer impreso -o en un blog viral- es lo que crea una figura estrella”. (5)

Las nuevas formas de difusión online de la arquitectura generan nuevos criterios para su valoración e impacto. Si anteriormente la calidad era el criterio principal, el mundo digital incluye otro tipo de estrategias basadas en parámetros y algoritmos, no siempre de fácil comprensión. Por eso, surge espontáneamente una pregunta sobre la dirección que está tomando la difusión de la disciplina y, en especial, de sus obras y los efectos sobre las nuevas generaciones de arquitectos. Una reflexión que Rafael Moneo ya planteó al reconocer la posibilidad de una cada vez mayor individualidad del proceso arquitectónico a menudo desligada de la historia. (6) Aunque también ha de reconocerse la retroalimentación producida por las imágenes a las cuales estamos sometidos a diario, generando un proceso de evolución y adaptación respecto a obras anteriores, según la teoría de "variant inflects model" sugerida por Dana Cuff. (7)

El presente estudio se enmarca en un trabajo de análisis más amplio acerca de la transformación de la difusión arquitectónica en los medios digitales. Su objetivo es comprender los cambios que se están produciendo en la forma de narración de la historia de la arquitectura del siglo xxi y, en particular, de las tendencias originadas por el incesante y progresivo uso de internet, $y$ su relación con los medios tradicionales. En particular, este texto utiliza las habituales herramientas de análisis y obtención de métricas de las redes para evidenciar el ecosistema de la difusión arquitectónica digital en la actualidad. Posteriormente, el ensayo analiza un perfil concreto activo en Facebook, FIG Projects, para resaltar el papel de las redes sociales en la diseminación de la arquitectura y evidenciar el papel del crítico o editor 'de antaño' frente a otros perfiles de mayor escala y estructura. Así se reconoce tanto el nuevo carácter de la difusión arquitectónica como el papel de las redes sociales en la misma.

The data collected provide very significant results to understand the current trend in architectural dissemination. A first interpretation shows the limited weight of architecture in the digital world, placing only 26 specialized media among the 100,000 most popular websites. A second reading places a clear preponderance of purely digital platforms with 21 media compared to only five online versions adapted from traditional media. (Fig. 7) This already limited number of magazines should even be reduced to just two magazines if only those specialized in architecture are considered -such as Architect, the magazine of the American Institute of Architects, and The Architect's Newspaper-, since the rest complement their contents with other related disciplines to suit a more general public.

Similarly, a dominant role of Anglo-Saxon media is also observed, both in the native online platforms and in the translation of printed publications to the digital medium. The institutional websites of some organizations such as the American Institute of Architects (AIA) or the Royal Institute of British Architects (RIBA) have even more visibility than most of the major traditional magazines. This implies a greater weight and, ultimately, impact of the professional organizations as compared to other dissemination models.

Another outcome is found in the authority of the conglomerate generated around ArchDaily as the most relevant digital repository in the field of architecture. (8) Since its launch more than a decade ago, ArchDaily has experienced continuous growth and is currently visited by more than 13 million people every month, publishing more than 4,000 projects per year -according to its own data-. The site has several versions in different languages to offer its content in a more specific form and to a wider audience: Spanish, Portuguese and Chinese. Moreover, the first language has three regional domains -ArchDaily Mexico, ArchDaily Colom- 
El impacto de los medios digitales en el ámbito de la arquitectura. Para comprender las tendencias actuales en la diseminación de la arquitectura se realiza un análisis comparativo entre los principales medios dedicados a la difusión de la misma. Al sustentarse gran parte de la difusión de hoy en día en la esfera digital, y considerando el permanente incremento de usuarios, (Figs. 4 y 5) se ha optado por centrar el análisis exclusivamente en el mundo digital.

La herramienta utilizada es Alexa.com, una de las aplicaciones más reconocidas para la medición del tráfico, la relevancia y el posicionamiento de las páginas web. El análisis se ha centrado tanto en los principales repositorios de arquitectura digitales como en las versiones online de los medios tradicionales más importantes. En concreto, el estudio ha valorado principalmente tres aspectos ofrecidos por Alexa en sus estadísticas: las visualizaciones diarias por visitante, el tiempo gastado diariamente por visitante $y$, sobre todo, el puesto en el ranking global basado en "el tráfico y la interacción global de la web en los últimos 90 días” como explica la propia web. (Fig. 6)

Los datos recopilados arrojan resultados muy esclarecedores para comprender la tendencia actual en la diseminación arquitectónica. Una primera interpretación muestra el escaso peso de la arquitectura en el mundo digital, al situar tan solo 26 medios especializados entre las 100.000 webs más populares. Una segunda lectura sitúa una clara preponderancia de las plataformas puramente digitales con 21 medios frente a solamente 5 versiones online adaptadas de los medios tradicionales. (Fig. 7) Este número debe incluso reducirse a dos únicas revistas si solo se consideran aquellas especializadas en arquitectura -como son Architect, la revista del American Institute of Architects, y The Architect's Newspaper-, puesto que el resto complementa sus contenidos con otras disciplinas afines para adaptarse a un público más general.

Del mismo modo, también se observa un papel dominante de los medios anglosajones, tanto en las plataformas nativas online como en la traslación
Fig. 4. Porcentaje de usuarios que afirman usar cada uno de los medios para acceder a contenido informativo (noticias). Hootsuite Report, julio 2020.

\section{ONLINE MEDIA}

(INC. SOCIAL MEDIA)
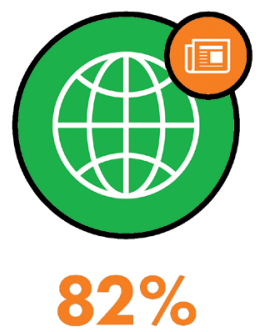

FEMALE: MALE:

$81 \% \quad 82 \%$
TELEVISION (BROADCAST \& CABLE)
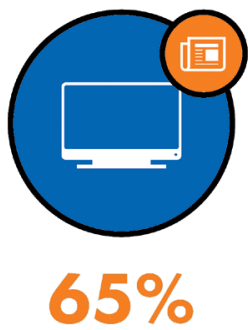

FEMALE: MALE:

$66 \% \quad 65 \%$
SOCIAL MEDIA (INC. MESSENGERS)
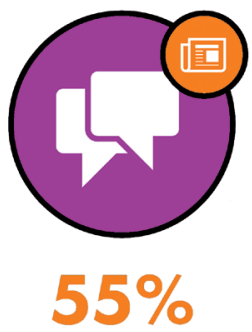

FEMALE: MALE:

$57 \% \quad 53 \%$
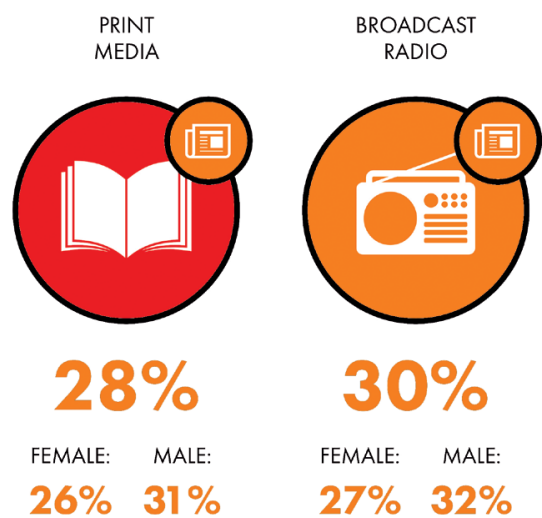
al medio digital de las publicaciones impresas. Las webs institucionales de algunas organizaciones como el American Institute of Architects (AIA) o el Royal Institute of British Architects (RIBA) tienen incluso más visibilidad que la mayoría de las revistas tradicionales más importantes. Ello implica un mayor peso y, en definitiva, impacto de las organizaciones colegiales frente a otros modelos de difusión.

Otro resultado de interés originado por el análisis se encuentra en la autoridad del conglomerado generado alrededor de ArchDaily como el repositorio digital -y, por lo tanto, el medio de difusión- más importante en el campo de la arquitectura. (8) Desde su aparición hace más de una década, ArchDaily ha experimentado un continuo crecimiento y actualmente es visitada por más de 13 millones de personas cada mes, publicando más de 4.000 proyectos al año, -según datos de la propia compañía-. La página cuenta con varias versiones en diferentes lenguas para ofrecer su contenido de una manera más específica y a un público más amplio: español, portugués y chino. A su vez, el primer idioma cuenta con tres páginas regionales -ArchDaily México, ArchDaily Colombia, y ArchDaily Perú- y una general para el mundo hispanoparlante bajo el nombre de Plataforma Arquitectura -en realidad, el conglomerado nació a partir de esta página, pero pronto creó su versión en inglés para ampliar su audiencia y difusión-. En total son siete páginas dedicadas principalmente a la difusión de la arquitectura y todas ellas están situadas entre las 20 webs más importantes del sector. El conglomerado ha sido recientemente adquirido por el portal suizo Architonic, un catálogo online de productos y materiales relacionados con la construcción, con el objetivo de complementarse y expandir todavía más su presencia en la red. (9)

Esta comparativa muestra un claro dominio de los medios nativos digitales frente a las adaptaciones de los medios tradicionales de difusión de contenido arquitectónico. Por un lado, debido a una mayor sintonía de los primeros con los usuarios nativos digitales. Y por otro, por una lenta y compleja adaptación o transición de los medios físicos de divulgación a la red, ya que en algunos casos las versiones digitales son aún simples plata-
Fig. 5. Número de personas que usan redes sociales activamente. Hootsuite Report, julio 2020.
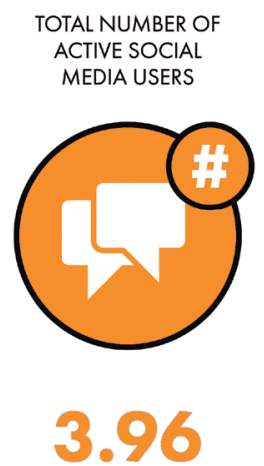

BILLION
SOCIAL MEDIA PENETRATION (USERS vs. TOTAL POPULATION*)
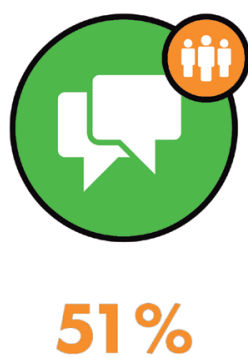

ANNUAL GROWTH IN THE TOTAL NUMBER OF SOCIAL MEDIA USERS
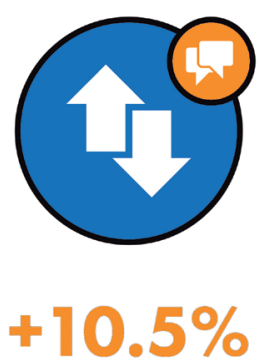

+376 MILLION
TOTAL NUMBER OF SOCIAL MEDIA USERS ACCESSING VIA MOBILE PHONES
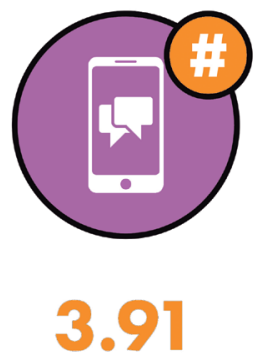

BILLION
PERCENTAGE OF TOTAL SOCIAL MEDIA USERS ACCESSING VIA MOBILE
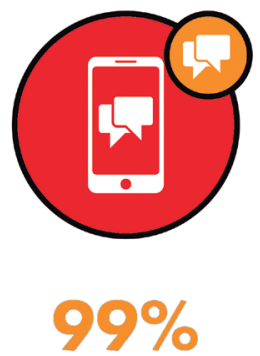


\begin{tabular}{|c|c|c|c|c|c|c|}
\hline \multirow[t]{2}{*}{ Web } & \multicolumn{2}{|c|}{ Puesto en Alexa } & \multicolumn{2}{|c|}{$\begin{array}{l}\text { Páginas diarias } \\
\text { visitadas por } \\
\text { usuario }\end{array}$} & \multicolumn{2}{|c|}{$\begin{array}{c}\text { Tiempo medio } \\
\text { diario por } \\
\text { visitante }\end{array}$} \\
\hline & $\begin{array}{c}\text { enero } \\
2020\end{array}$ & $\begin{array}{l}\text { sept. } \\
2020\end{array}$ & $\begin{array}{c}\text { enero } \\
2020\end{array}$ & $\begin{array}{l}\text { sept. } \\
2020\end{array}$ & $\begin{array}{c}\text { enero } \\
2020\end{array}$ & $\begin{array}{l}\text { sept. } \\
2020\end{array}$ \\
\hline Archdaily.com & 2.697 & 1.768 & 6.24 & 7.24 & $5: 44$ & $4: 30$ \\
\hline Dezeen.com & 4.666 & 4.401 & 2.1 & 2.1 & $2: 38$ & $2: 26$ \\
\hline Designboom.com & 7.417 & 7.893 & 3.29 & 4.3 & $3: 42$ & 4:02 \\
\hline Plataformaarqutiectura.cl & 6.097 & 8.242 & 3.6 & 3.2 & $3: 45$ & $3: 27$ \\
\hline Architecturaldigest.com ${ }^{*}$ & & 8.505 & & 1.48 & & $1: 57$ \\
\hline Dwell.com* & & 10.975 & & 4.9 & & $2: 27$ \\
\hline Archinect.com & 21.431 & 18.732 & 12 & 18 & $6: 08$ & $7: 16$ \\
\hline Archdaily.mx & & 25.167 & & 4.2 & & $2: 05$ \\
\hline Archello.com & & 27.635 & & 14 & & $32: 58$ \\
\hline Archdaily.cn & & 29.098 & & 4.4 & & $3: 14$ \\
\hline Architizer.com & & 31.051 & & 3.1 & & $2: 46$ \\
\hline Archilovers.com & & 35.297 & & 3.3 & & 3:13 \\
\hline Wallpaper.com* & & 50.097 & & 2.6 & & $1: 30$ \\
\hline Divisare.com & & 56.765 & & 2.3 & & $2: 24$ \\
\hline Gooood.cn & & 57.557 & & 3.9 & & $4: 11$ \\
\hline Archdaily.pe & & 59.279 & & 1.5 & & $1: 59$ \\
\hline Afasiaarchzine.com & & 61.463 & & 5.6 & & $1: 35$ \\
\hline Archdaily.co & & 62.233 & & 6.2 & & $2: 24$ \\
\hline Hicarquitectura.com & & 62.784 & & 1.4 & & $2: 02$ \\
\hline Archdaily.com.br & & 64.638 & & 3.7 & & $3: 25$ \\
\hline Arch20.com & & 66.484 & & 1.6 & & $2: 04$ \\
\hline Arq.com.mx & & 67.933 & & 3.4 & & $3: 40$ \\
\hline Architectmagazine.com & & 68.859 & & 1.4 & & $1: 47$ \\
\hline AIA.org** & & 83.939 & & 2.7 & & $3: 00$ \\
\hline Arqa.com & & 84.557 & & 1.5 & & $0: 32$ \\
\hline Archcollege.com & & 87.266 & & 2.4 & & $1: 53$ \\
\hline Architecture.com ${ }^{\star \star}$ & & 88.134 & & 3.2 & & $2: 23$ \\
\hline Archpaper.com* & & 95.115 & & 1.4 & & $1: 27$ \\
\hline
\end{tabular}

Fig. 6. Estadísticas ofrecidas por Alexa para las versiones digitales de los principales medios tradicionales impresos ( 9 de septiembre de 2020).

bia, and ArchDaily Peru- and a general page for the Spanish-speaking world under the name of Plataforma Arquitectura -the conglomerate started with this page, but soon created its English version to expand its audience and dissemination-. To resume, there are seven pages dedicated mainly to the dissemination of architecture and all of them are ranked among the twenty most important websites in the sector. The conglomerate has recently been acquired by the Swiss portal Architonic, an online catalog of products and materials related to construction, to complement each other and further expand their presence on the web. (9)

This comparison shows a clear dominance of native digital media versus traditional media adaptations of architectural content. On the one hand, due to a greater harmony of the former with the digital native users. On the other hand, the digital versions are still simple platforms for digital sales or subscription due to an often slow and complex adaptation or transition of the physical media to the network. (10) This last aspect has meant that, in most cases, the traditional media have lost their position of dominance and relevance in the physical world, something that can only be recovered by rethinking their dissemination model. The speed of transformations in the digital world has undoubtedly favored this digital gap between traditional and digital media, although the same statistics point to a slight upward trend for media adapted to the network.

The Rise of Social Networks as a Means of Disseminating Knowledge. The current trend in the web is to reinforce the 'shared knowledge' concept. In the last five years, daily mobile internet access has almost doubled to 3 hours and 14 minutes -currently 48 percent of total daily internet use is done only through this device- and the time spent daily on social networks has increased to 2 hours and 16 minutes. (11) This trend displaces the business model to an in- 
formas de venta o suscripción digital. (10) Este último aspecto ha supuesto que, en la mayoría de los casos, los medios tradicionales hayan perdido la posición de dominio y relevancia que atesoraban en el mundo físico, algo que puede ser recuperado solo replanteando su modelo de difusión. La velocidad de las transformaciones en el mundo digital ha favorecido sin duda esta brecha digital entre medios tradicionales y digitales, aunque las mismas estadísticas señalan una leve tendencia al alza a favor de los medios adaptados a la red.

El auge de las redes sociales como medio de diseminación del conocimiento. La tendencia actual en la red se inclina a reforzar el concepto shared knowledge. En los últimos cinco años casi se ha duplicado el acceso diario a internet con el móvil hasta las 3 horas y 14 minutos -en la actualidad el 48\% del uso diario total de internet se realiza únicamente a través de este dispositivo- y ha aumentado el tiempo empleado a diario en las redes sociales hasta las 2 horas y 16 minutos. (11) Esta tendencia está desplazando el modelo empresarial a una cada vez más decidida presencia en las redes sociales, en detrimento de las páginas web. De ahí que la presencia en este tipo de redes sea cada vez más importante tanto para el posicionamiento como para la difusión en general de contenidos. (Fig. 8) Esta nueva dirección implica una variación en el uso de internet desde plataformas unidireccionales o estáticas a otras bidireccionales o más participativas y dinámicas con la cocreación de contenidos y la condivisión de experiencias. Es decir, desde las páginas webs hacia las redes sociales.

Dicha tendencia se confirma al observar la existencia de perfiles de las webs de difusión antes analizadas en las principales redes sociales. En muchos casos sus perfiles siguen demostrando un dominio similar a las webs en cuanto a sus altos números de seguidores o de contenidos publicados. No obstante, la cantidad de seguidores no siempre está alineada con la interacción. Es decir, un gran número de usuarios no garantiza un uso activo del contenido publicado y este aspecto es clave en el nuevo medio. Los algoritmos de las redes sociales favorecen aquellas publicaciones propensas a recibir más participa-

\begin{tabular}{|l|c|c|c}
\hline \multicolumn{1}{|c|}{ Página Web } & $\begin{array}{c}\text { Puesto en } \\
\text { Alexa }\end{array}$ & $\begin{array}{c}\text { Páginas diarias } \\
\text { visitadas por } \\
\text { usuario }\end{array}$ & $\begin{array}{c}\text { Tiempo medio } \\
\text { diario por } \\
\text { visitante }\end{array}$ \\
\hline Architectsjournal.co.uk & 117.376 & 1.3 & $1: 15$ \\
Domusweb.it & 162.248 & 2.3 & $2: 14$ \\
Architecturalrecord.com & 170.450 & 1.3 & $1: 25$ \\
Metalocus.com & 198.888 & 2 & $1: 25$ \\
Frameweb.com & 228.658 & 1.6 & $1: 28$ \\
Metropolismag.com & 256.586 & 2.0 & $1: 41$ \\
Architectural-review.com & 337.391 & 1.9 & $1: 26$ \\
Azuremagazine.com & 405.000 & 1.4 & $1: 22$ \\
Arquitecturaviva.com & 432.472 & 1 & $2: 18$ \\
Arquine.com & 449.816 & 1.1 & $1: 24$ \\
Detail.de & 557.987 & 2 & $2: 12$ \\
Bawuwelt.de & 924.734 & 2 & $2: 56$ \\
\hline
\end{tabular}

Fig. 7. Las redes sociales más usadas alrededor del mundo; basado en perfiles activos (en millones). Hootsuite Report (julio 2020). 
ción fomentando todavía más la visibilidad de este contenido, siempre en pos de aumentar el tiempo consumido en las plataformas. De ahí que la fidelidad de los seguidores y el número de comentarios juegue un papel crucial para definir la relevancia en las plataformas participativas. Para entender mejor las posibilidades que ofrecen las redes sociales frente a los portales anteriormente descritos, se plantea el estudio de un perfil específico, aprovechando la posibilidad de realizar una entrevista no estructurada con sus editores que arroja una mejor comprensión y contextualización de su funcionamiento.

En el panorama actual de las redes sociales existen numerosos perfiles creados específicamente para las mismas -sin tener el apoyo de una web de referencia- que consiguen mayores interacciones que las grandes plataformas. Un ejemplo de esta tendencia es el perfil FIG Projects en Facebook, gestionado por Fabrizio Gallanti y Francisca Insulza. (Fig. 9) Los números de este perfil son muy inferiores en cuanto a seguidores y publicaciones semanales frente a los perfiles sociales de las grandes plataformas, como ArchDaily, Designboom o Dezeen. Aun así, FIG Projects tiene una interacción semanal medida en reacciones, comentarios o veces que se compartió, similar a los grandes perfiles e incluso, en ocasiones, muy superior. (Fig. 10)

El valor del perfil FIG Projects, según los propios editores, se fundamenta principalmente en varios aspectos. Por un lado, son ellos mismos quienes buscan el contenido en múltiples fuentes y quienes lo conectan siguiendo una lógica editorial -por temas, autores, épocas...-. Esto les permite tomar una posición crítica independiente, mientras que en los grandes portales predomina el contenido publicado sin ningún tipo de búsqueda, edición o criterio editorial. Otro aspecto clave de FIG Projects es la heterogénea mezcla de arquitectura de diferentes periodos históricos y contextos, en lugar de prestar atención exclusiva a la arquitectura mainstream contemporánea como suele suceder en las grandes plataformas. (12)

Existen perfiles con éxito similar a FIG Projects y todos ellos comparten características similares, aunque se sitúen en distintas redes sociales. Nor-
Fig. 8. Perfil FIG Projects. Facebook, 2020.

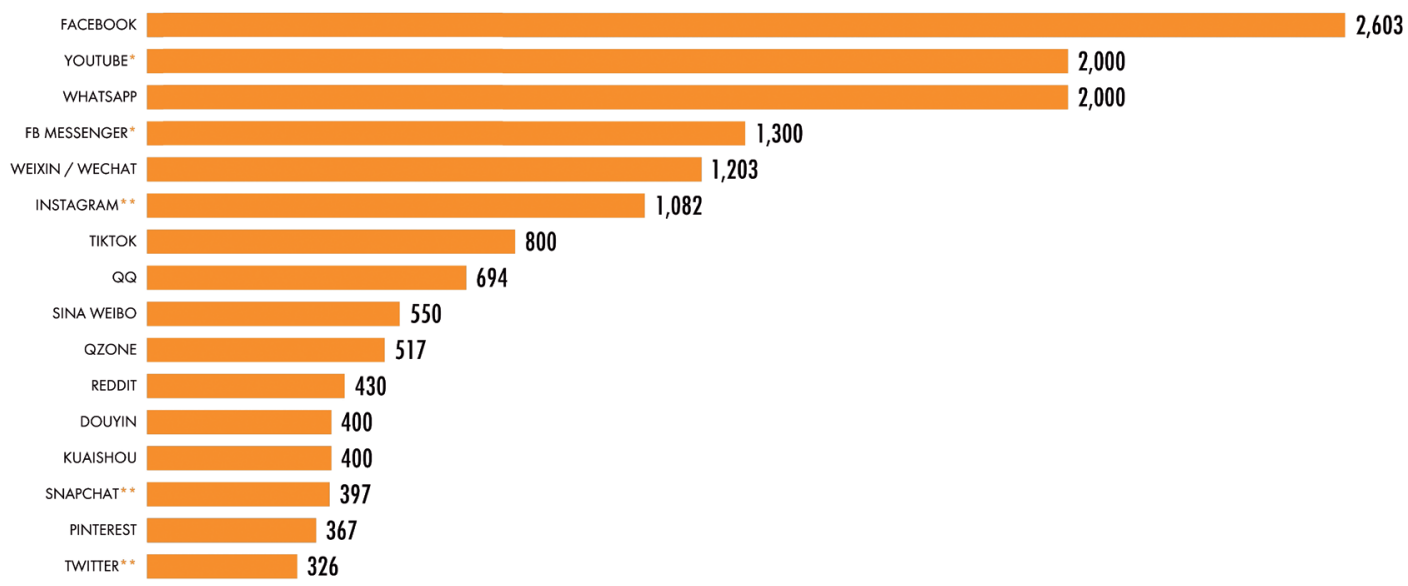




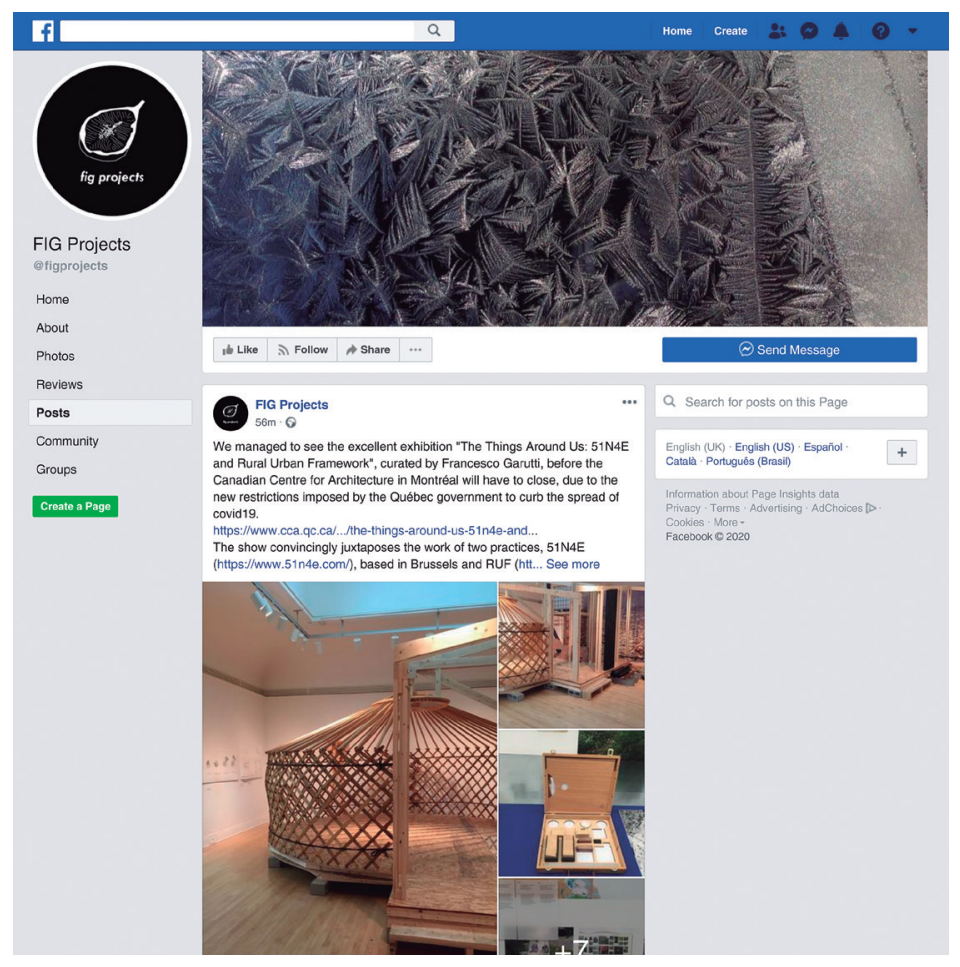

malmente cuentan con una infraestructura mínima formada por una o dos personas que gestionan su imagen bajo motivaciones personales no lucrativas. Esta motivación genera una mayor dedicación y cuidado en la preparación de contenidos, tanto en su calidad como en el seguimiento e interacción con el público y sus reacciones. El resultado es un mayor entendimiento de la red social utilizada y por lo tanto una perfecta adecuación de los contenidos a publicar. Si bien el enfoque monocanal -en el
Fig. 9. Rendimiento de perfiles arquitectónicos en Facebook. Crecimiento y fidelidad de usuarios activos durante los últimos meses. Facebook 2020.

creasingly strong presence in social networks to the detriment of websites. Hence, this type of network is increasingly important for positioning and disseminating content in general. (Fig. 8) This new direction implies a variation in the use of the internet from unidirectional or static platforms to other more participative and dynamic ones with the cocreation of contents and sharing for experiences. That is, from websites to social networks.

This trend is confirmed by the existence of profiles of the websites previously analyzed in the main social networks. Their profiles continue to show a similar domain in terms of their high numbers of followers or published content in many cases. However, the number of followers is not always aligned with the interaction. Many users do not guarantee an active use of the published content, which is vital in the new medium. The algorithms of social networks favour those publications that are prone to receive more participation, promoting, even more, the visibility of this content, always to increase the time spent on the platforms. Hence, the loyalty of the followers and the number of comments play a crucial role in defining the relevance on the participatory platforms. To better understand the possibilities offered by social networks compared to the portals described above, we propose the study of a specific profile, taking advantage of the possibility of conducting an unstructured interview with their editors that provides a better understanding and contextualization of their operation.

In today's social network landscape, there are many profiles explicitly created for social networks -without the support of a reference website- that achieve more significant interactions than large platforms. An example of this trend is the FIG Projects profile on Facebook, managed by Fabrizio Gallanti and Francisca Insulza. (Fig. 9) The numbers of this profile are 


\begin{tabular}{|c|c|c|c|c|c|c|c|c|}
\hline Page & & & Total $\mathrm{Pa}$ & age Likes & From last week & Posts This Week & Engager & ment This Week \\
\hline 1 & & ArchDaily & $2.3 m$ & $\square$ & $0 \%$ & 125 & $182.9 \mathrm{~K}$ & 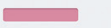 \\
\hline 2 & 1 & designboom & $1.2 \mathrm{~m}$ & $\square$ & $0 \%$ & 89 & $52.4 \mathrm{~K}$ & $\square$ \\
\hline 3 & de & Dezeen & $1.1 \mathrm{~m}$ & $\square$ & $0 \%$ & 156 & $201.8 \mathrm{~K}$ & $\square$ \\
\hline 4 & domus & Domus & $703.7 \mathrm{~K}$ & $\square$ & $0 \%$ & 60 & $65.1 \mathrm{~K}$ & $\square$ \\
\hline 5 & & The Architect's Newspa... & $677.2 \mathrm{~K}$ & $\square$ & $0 \%$ & 42 & $4.5 \mathrm{~K}$ & 1 \\
\hline 6 & ) & FIG Projects & $54.4 \mathrm{~K}$ & 1 & $\Delta 5.7 \%$ & 20 & $255.4 \mathrm{~K}$ & $\square$ \\
\hline Page & & & Total Pa & age Likes & From last week & Posts This Week & Engager & ment This Week \\
\hline 1 & & ArchDaily & $2.3 m$ & 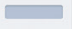 & $0 \%$ & 96 & $162.2 \mathrm{~K}$ & 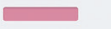 \\
\hline 2 & & designboom & $1.2 \mathrm{~m}$ & $\square$ & $0 \%$ & 100 & $51.3 \mathrm{~K}$ & - \\
\hline 3 & deen & Dezeen & $1.1 \mathrm{~m}$ & $\square$ & $0 \%$ & 151 & $200.5 \mathrm{~K}$ & 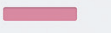 \\
\hline 4 & domus & Domus & $703.7 \mathrm{~K}$ & $\square$ & $0 \%$ & 66 & $66.3 \mathrm{~K}$ & $\square$ \\
\hline 5 & & The Architect's Newspa... & $677.2 \mathrm{~K}$ & $\square$ & $0 \%$ & 44 & $4.6 \mathrm{~K}$ & 1 \\
\hline 6 & 3 & FIG Projects & $55.2 \mathrm{~K}$ & 1 & $\Delta 5.6 \%$ & 23 & $210.1 \mathrm{~K}$ & $\square$ \\
\hline Page & & & Total Pa & age Likes & From last week & Posts This Week & Engage & ment This Week \\
\hline 1 & & ArchDaily & $2.3 \mathrm{~m}$ & $\square$ & $0 \%$ & 131 & $179.4 K$ & \\
\hline 2 & & designboom & $1.2 \mathrm{~m}$ & $\square$ & $0 \%$ & 91 & $55.2 \mathrm{~K}$ & - \\
\hline 3 & en & Dezeen & $1.1 \mathrm{~m}$ & $\square$ & $0 \%$ & 158 & $186.2 \mathrm{~K}$ & $\square$ \\
\hline 4 & domus & Domus & $703.7 \mathrm{~K}$ & $\square$ & $0 \%$ & 61 & $66.6 \mathrm{~K}$ & $\square$ \\
\hline 5 & & The Architect's Newspa... & $677.2 \mathrm{~K}$ & $\square$ & $0 \%$ & 42 & $4.2 \mathrm{~K}$ & I \\
\hline 6 & & FIG Projects & $53.9 \mathrm{~K}$ & 1 & $\Delta 6.2 \%$ & 20 & $236.1 \mathrm{~K}$ & $\square$ \\
\hline Page & & & Total $\mathrm{Pa}$ & age Likes & From last week & Posts This Week & Engage & ment This Week \\
\hline 1 & & ArchDaily & $2.3 \mathrm{~m}$ & $\square$ & $0 \%$ & 113 & $177.6 \mathrm{~K}$ & $\square$ \\
\hline 2 & & designboom & $1.2 \mathrm{~m}$ & $\square$ & $0 \%$ & 94 & $52 k$ & $\square$ \\
\hline 3 & de & Dezeen & $1.1 \mathrm{~m}$ & $\square$ & $0 \%$ & 157 & $202.6 \mathrm{~K}$ & 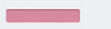 \\
\hline 4 & domus & Domus & $703.7 \mathrm{~K}$ & 담 & $0 \%$ & 66 & $66.2 \mathrm{~K}$ & 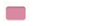 \\
\hline 5 & & The Architect's Newspa... & $677.2 \mathrm{~K}$ & $\square$ & $0 \%$ & 42 & $4.6 \mathrm{~K}$ & । \\
\hline 6 & $y$ & FIG Projects & $54.8 \mathrm{~K}$ & 1 & $\Delta 5.5 \%$ & 24 & $227.6 \mathrm{~K}$ & \\
\hline
\end{tabular}

Fig. 10. Ejemplo de feed en Facebook. Noticias, sugerencias, publicaciones profesionales y sociales se entremezclan para mantenernos consumiendo contenido. 
caso de FIG Projects a través de Facebook- podría entenderse como una limitación para su difusión, en realidad fortalece la impresión de credibilidad que se transmite al aunar todas las interacciones en un solo portal y tener una atención más cercana. (13) Es decir, se retoma la figura tradicional de crítico/editor, pero rompiendo la distancia con el lector.

Si las webs monotemáticas han posibilitado la 'democratización de la visibilidad', por la que pequeñas plataformas y/o arquitectos pueden optar a una gran visibilidad anteriormente solo accesible a grandes estructuras, las redes sociales han favorecido la 'democratización de la opinión' ya que en ellas cualquier persona puede elevar su opinión o crítica tuits, posts o comentarios- y ser considerada globalmente hasta hacerse relevante o viral. En otras palabras, las redes sociales han convertido al usuario en productor, editor y crítico de arquitectura -a menudo a la vez- y esto está cambiando las reglas tradicionales de difusión de la arquitectura.

Nuevas reglas para un nuevo medio. El ecosistema para la difusión de la arquitectura ha crecido con la inclusión de los canales digitales, y su proliferación y relevancia los está convirtiendo en el lugar de almacenamiento del conocimiento arquitectónico en la era actual. Esto ha supuesto una inclusión de nuevos actores y la modificación del papel del usuario, quien ahora ya no ejerce únicamente un rol pasivo, sino que también es artífice de su creación y, sobre todo, demanda inmediatez, ubicuidad y facilidad de acceso a todo tipo de contenidos.

Un ejemplo es la cada vez mayor brecha existente entre los medios de comunicación de arquitectura convencionales y los contenidos demandados por el usuario en la red. Jaume Prat señala:

"Mientras en las publicaciones cultas triunfa el postmodernismo en las redes se impone lo matérico ahí quedan iniciativas como el Lunes Brutalista, el Miércoles Enladrillado y tantos perfiles que, lentamente, sub-

much lower in terms of followers and weekly publications compared to the social profiles of larger platforms, such as the already mentioned ArchDaily, Designboom or Dezeen. Still, FIG Projects has a weekly interaction measured in reactions, comments or times shared, similar to the large profiles and sometimes even much higher. (Fig. 10)

The value of the FIG Projects profile, according to the editors themselves, is mainly based on several aspects here resumed. First, they search for content from multiple sources and connect it according to an editorial logic -by subject, author, period...-. This allows them to take an independent critical position, while in the large portals the published content predominates without any type of search, editing or editorial criteria. Another key aspect of FIG Projects is the heterogeneous mix of architecture from different historical periods and contexts, rather than paying exclusive attention to contemporary mainstream architecture as is often the case with large platforms. (12)

There are successful profiles similar to FIG Projects and they all share similar characteristics even though they are active on different social networks. They usually have a minimum infrastructure consisting of one or two people who manage their image under personal and non-profit motivations. These motivations generate greater dedication and care in the preparation of content, both in its quality and in the monitoring and interaction with the public and its reactions. The result is a better understanding of the social network used and, therefore, a greater optimization of the contents to be published. While the single-channel approach -in the case of FIG Projects through Facebook- could be understood as a limitation for its dissemination, it actually strengthens the impression of credibility conveyed by bringing all interactions 
vierten el canon ampliándolo [...] La capacidad de cruzar informaciones del que busca estas arquitecturas, la capacidad de comisariar contenidos de algunos usuarios ha desplazado las publicaciones del centro -o de la exclusiva- a ser, en el mejor de los casos, un agente más". (14)

El sexto informe DOMO 'Data Never Sleeps' estima que en 2020 se creará en internet $1.7 \mathrm{Mb}$ de contenido cada segundo por cada persona en la Tierra y se subirán 49.000 fotos por minuto a Instagram. (15) Entre sus publicaciones diarias, ArchDaily genera una media de entre 10 y 15 proyectos al día, lo que implica emplear nuevas estrategias de comunicación para obtener la máxima repercusión. De ahí, que se recurra al medio que se ha entendido más rápido para transmitir una idea: la imagen. Esta pasa de ser una representación de la idea proyectual a ser un fin, una herramienta no siempre fiel al original realizada con el objetivo de destacar sobre la competencia y por ende ser visitado por los usuarios digitales. Este fenómeno, que como vimos al comienzo de este artículo no es en absoluto nuevo, requiere de una importante capacidad analítica para poder analizar y comentar correctamente un proyecto arquitectónico, y no exclusivamente la imagen visualizada. Los efectos en la evolución de la arquitectura, especialmente en las generaciones nativas digitales más jóvenes son por lo tanto relevantes.

Esta sobreproducción de imágenes pensadas para atraer nuestra atención requiere hoy más que nunca ser complementada, para ofrecer mayor rigor y una correcta comprensión y valoración del proyecto arquitectónico. La paulatina desaparición de editores y críticos, antaño responsables de la selección y análisis de los proyectos de arquitectura publicados, requiere reflexionar sobre cómo la democratización de las opiniones pueda sustituir esta labor. Generalmente, en los procesos shared knowledge el análisis y comprensión de una obra se genera de forma coral y altruista, independientemente de rangos y roles que se desdibujan detrás de alias no siempre reconocibles, siendo accesibles de forma libre para todos los usuarios de la red. La puesta en común y la discusión del conocimiento individual y

together in a single portal that receives closer attention. (13) In other words, the traditional figure of the critic/editor is recovered but breaking the distance with the reader.

If the monothematic websites have made possible the 'democratization of visibility', by which small platforms and/or architects can opt for great visibility previously only accessible to large structures, social networks have favored the 'democratization of opinion' since in them anyone can raise their opinion or criticism -tweets, posts or comments- and be considered globally until they become relevant or viral. In other words, social networks have turned the user into a producer, editor and critic of architecture -often at the same time- and this is changing the traditional rules of architectural dissemination.

New Rules for a New Medium. The ecosystem for the diffusion of architecture has grown with the inclusion of digital channels. Their proliferation and relevance are making them the storage place for architectural knowledge in the current era. This has meant the inclusion of new actors and the modification of the role of the user, who now no longer plays only a passive role, but is also the maker of its creation and, above all, demands immediacy, ubiquity and ease of access to all types of content.

One example is the growing gap between conventional architectural media and the content demanded by the user on the web. Jaume Prat points out:

"And so, it goes on: while postmodernism reigns supreme in the sophisticated journals, it's a much more material, truer 


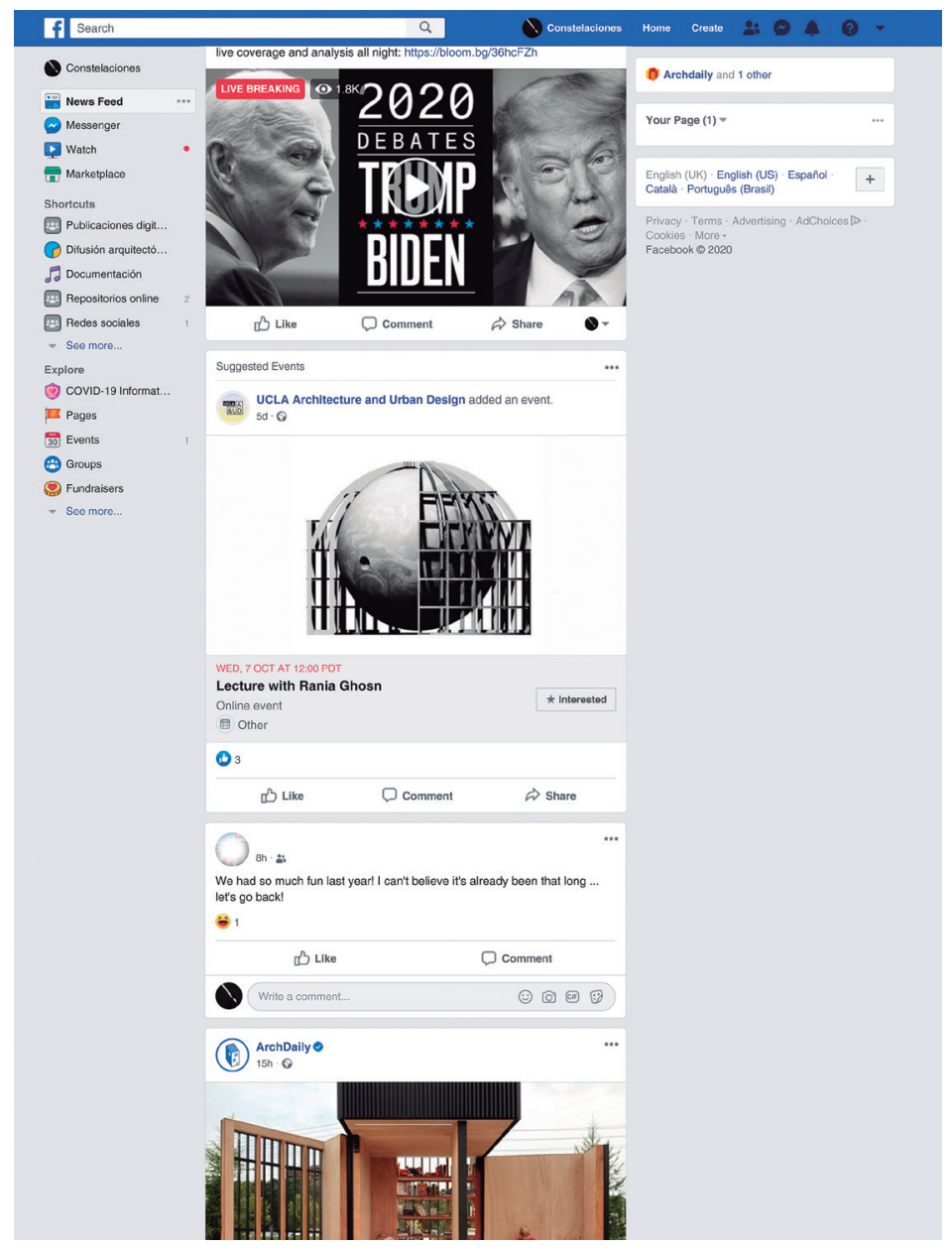

Fig. 11. Ejemplo de feed en Facebook. Noticias, sugerencias, publicaciones profesionales y sociales se entremezclan para mantenernos consumiendo contenido.

to life architecture that prevails in the social media. There we find initiatives like Lunes Brutalista -Brutalist Monday-, Miércoles Enladrillado -Brickwork Wednesday- and countless profiles [...] that slowly subvert the conventional canon by expanding its scope [...] The capacity of some social media users to exchange information with seekers of these architectures and to curate their own content has pushed journals and publications off their pedestal of pre-eminence -or exclusivity-, turning them, at best, into just another resource". (14)

The sixth DOMO report 'Data Never Sleeps' estimates that by $20201.7 \mathrm{MB}$ of content will be created on the internet every second by every person on Earth, and 49,000 photos per minute will be uploaded to Instagram. (15) Among its daily publications, ArchDaily generates an average of 10-15 projects per day, which requires employing new communication strategies to achieve maximum impact. As a result, they use the medium that has been most quickly understood to convey an idea: image. This goes from being a representation of the design idea to being an end, a tool not always faithful to the original made with the aim of standing out from the competition and therefore being visited by digital users. This phenomenon, which is not new as we saw at the beginning of this article, requires an important analytical capacity to correctly analyze and comment on an architectural project, and not exclusively on the visualized image. The effect on the evolution of architecture, especially on the younger digital native generations is therefore relevant.

This overproduction of images designed to attract our attention requires today more than ever to be complemented, to offer greater rigor and a correct understanding and assessment of the architectural project. The gradual disappearance of editors 
de las experiencias personales relacionadas sobre un tema, actual o pasado, ofrece la forma de superar la simple difusión de un proyecto, enriqueciendo el conocimiento global con aportaciones múltiples y variadas, a las cuales puede acceder cualquiera, incluso de forma accidental. Este valor añadido difícilmente puede obtenerse con medios tradicionales, a los que no sustituye sino complementa.

Es por lo tanto necesario fomentar la capacidad de discernimiento y crítica en las escuelas como competencia indispensable para el uso de los nuevos medios de difusión. De una forma parecida en la que el escepticismo y el contraste deshacen las fake news, la valoración crítica más allá de lo visual se torna primordial en el contexto arquitectónico de las redes sociales. La limitada interacción que experimentan los canales de las grandes plataformas de difusión en relación con el número de seguidores, contrasta con el interés del público para los perfiles más especializados que reciben una mayor interacción, y por ende generan mayor riqueza de contenidos.

Seguramente asistiremos pronto a un cambio en las políticas de gestión de muchos portales de arquitectura para fomentar aún más el sentimiento de comunidad, lo que aportará valor añadido a la experiencia arquitectónica online. La rapidez con la cual se disfrutan y fagocitan los proyectos publicados hace sin embargo desear una reflexión sobre cuál será el mejor foro para realizar y seguir el análisis y la crítica de los proyectos, en los cuales el usuario podrá optar por seguir análisis más rigurosos y contrastados o simplemente quedarse en la superficialidad e inmediatez de la imagen. (Fig. 11)

and critics, once responsible for selecting and analysing published architectural projects, requires reflection on how the democratization of opinions can replace this work. Generally, in shared knowledge processes, the analysis and understanding of a work is generated in a choral and altruistic way, independently of ranges and roles that are blurred behind not always recognizable aliases, being freely accessible to all network users. Sharing and discussing individual knowledge and related personal experiences on a topic, current or past, offers a way to overcome the simple diffusion of a project, enriching global knowledge with multiple and varied contributions, which can be accessed by anyone, even accidentally. This added value can hardly be obtained with traditional means, which it does not replace but complements.

Therefore, it is necessary to foster the capacity for discernment and criticism in schools as an indispensable competence for the use of new media. In a similar way in which skepticism and contrast undo the fake news, critical evaluation beyond the visual becomes primordial in the architectural context of social networks. The limited interaction experienced by the channels of the large broadcasting platforms in relation to the number of followers contrasts with the interest of the public for more specialized profiles that receive greater interaction, and therefore generate a greater wealth of content.

We will surely soon see a change in the management policies of many architectural portals to further foster a sense of community, which will add value to the online architectural experience. The speed with which the published projects are enjoyed and phagocytized makes us want to reflect on what will be the best forum to carry out and follow the analysis and criticism of the projects, in which the user will be able to choose a more rigorous and contrasting analysis or simply remain in the superficiality and immediacy of the image. (Fig. 11) 


\section{REFERENCIAS}

1. COLOMINA, B. Privacidad y publicidad. La arquitectura moderna como medio de comunicación de masas. Segunda Ed. Murcia: Centro de Documentación y Estudios Avanzados de Arte Contemporáneo, 2010, p. 10. 2. NAEGELE, D. Seeing what is not there yet: Le Corbusier and the architectural space of photographs. En: International Conference INTER-Photography and Architecture. [en línea], Pamplona: Universidad de Pamplona, 2016 [consulta: 20.09.2020]. Disponible en: https://lib.dr.iastate. edu/arch_conf/90

3. BOHIGAS, O. Hacia una arquitectura menos moderna. En: Contra una arquitectura adjetivada. Barcelona: Editorial Seix Barral, 1969, pp. 39-56. 4. SAINZ, J. Últimas monografías de arquitectura española, Arquitectura Viva, n. 10, 1990, pp. 42-43.

5. MAAS, W.; MADRAZO, F.; HULSMAN, B. (eds). Copy Paste - The badass Architectural Copy Guide. Amsterdam: Nai010 Publishers, 2017, p. 150.

6. MONEO, R. Soane Annual Lecture [en línea]. 2017 [consulta: 23.09.2020]. Disponible en: https://www.arquitecturaviva.com/Fmedia/ FDocumentos/moneo_soane_lecture.pdf

7. CUFF, D. Introduction: Architecture's double-bind. En: CRYSLER, C.G.; CAIRNS, S.; HEYNEN, H. (eds.). The Sage Handbook of Architectural Theory. London: SAGE Publications, 2012, pp. 385-392.

8. CIMADOMO, G.; GARCÍA RUBIO, R.; SHAHDADPURI ASWANI, V. Towards a (new) Architectural History for a Digital Age. Archdaily as a dissemination tool for architectural knowledge. En: Criticall II International Conference on Architectural Design \& Criticism. Madrid: Universidad Politécnica de Madrid, 2018, pp. 64-73.

9. ROJAS, I. Architonic compra ArchDaily para crear la mayor plataforma de arquitectura en línea. Lexlatin [en línea], 2020 [consulta: 28.09.2020]. Disponible en: https://lexlatin.com/noticias/suiza-architonic-chilenaarchdaily-se-unen-crean-mayor-plataforma-arquitectura-en 10. Entrevista (no publicada) de Cimadomo, G. a Aurora Fernández Per $(\mathrm{A}+\mathrm{T})$ en el marco de la primera edición del curso online de la Fundación General de la Universidad de Málaga "Escribir arquitectura: pautas y criterios", 2011.

11. Informe HOOTSUITE, We Are Social. Digital Report 2019 [en línea]. [consulta: 28.09.2020]. Disponible en: https://wearesocial.com/globaldigital-report-20191A

12. Entrevista de los autores a los editores de FIG Projects (septiembre 2020).

13. SCHIANCHI, P. Architecture on the web. A critical approach to communication. Padova: Libreria universitaria, 2014.

14. PRAT, J. El arquitecto está desnudo. Fundación Arquia Blog [en línea], 2019 [consulta: 2.01.2020]. Disponible en: https://blogfundacion. arquia.es/2019/12/el-arquitecto-esta-desnudo/

15. Sexto informe DOMO 'Data Never Sleeps'. Disponible en: https:// www.domo.com/learn/data-never-sleeps-6

\section{REFERENCES}

1. COLOMINA, B. Privacidad y publicidad. La arquitectura moderna como medio de comunicación de masas. Second Ed. Murcia: Centro de Documentación y Estudios Avanzados de Arte Contemporáneo, 2010, p. 10. 2. NAEGELE, D. Seeing what is not there yet: Le Corbusier and the architectural space of photographs. In: International Conference INTER -Photography and Architecture [online]. Pamplona: Universidad de Pamplona, 2016 [Accessed 20 September 2020]. Available at: https://lib. dr.iastate.edu/arch_conf/90

3. BOHIGAS, 0. Hacia una arquitectura menos moderna. In: Contra una arquitectura adjetivada. Barcelona: Editorial Seix Barral, 1969, pp. 39-56. 4. SAINZ, J. Últimas monografías de arquitectura española, Arquitectura Viva, n. 10, 1990, pp. 42-43.

5. MAAS, W., MADRAZO, F.; HULSMAN, B. (eds). Copy Paste - The badass Architectural Copy Guide. Amsterdam: Nai010 Publishers, 2017, p. 150. 6. MONEO, R. Soane Annual Lecture [online]. 2017 [Accessed 23

September 2020]. Available at: https://www.arquitecturaviva.com/Fmedia/ FDocumentos/moneo_soane_lecture.pdf

7. CUFF, D. Introduction: Architecture's double-bind. In: CRYSLER, C.G.; CAIRNS, S.; HEYNEN, H. (eds.). The Sage Handbook of Architectural Theory. London: SAGE Publications, 2012, pp. 385-392.

8. CIMADOMO, G.; GARCÍA RUBIO, R.; SHAHDADPURI ASWANI, V. Towards a (new) Architectural History for a Digital Age. Archdaily as a dissemination tool for architectural knowledge. In: Criticall III International Conference on Architectural Design \& Criticism. Madrid: Universidad Politécnica de Madrid, 2018, pp. 64-73.

9. ROJAS, I. Architonic compra ArchDaily para crear la mayor plataforma de arquitectura en línea. Lexlatin [online], 2020 [Accessed 28 September 2020]. Available at: https://lexlatin.com/noticias/suiza-architonic-chilenaarchdaily-se-unen-crean-mayor-plataforma-arquitectura-en

10. (Unpublished) Interview with Aurora Fernández Per $(A+T)$ held by Guido Cimadomo in the frame of the first edition of the online course 'Escribir arquitectura: pautas y criterios', Fundación General de la Universidad de Málaga, 2011.

11. HOOTSUITE, We Are Social. Digital Report 2019 [online]. [Accessed 28 September 2020]. Available at: https://wearesocial.com/global-digitalreport-20191A.

12. Interview with Fabrizio Galanti and Francisca Insulza, editors of FIG Projects, held by the authors (September, 2020).

13. SCHIANCHI, P. Architecture on the web. A critical approach to communication. Padova: Libreria universitaria, 2014.

14. PRAT, J. The architect has no clothes on! In: Fundación Arquia Blog. [online], 2019 [Accessed 2 January 2020]. Available at: https:// blogfundacion.arquia.es/en/2019/12/the-architect-has-no-clothes-on/ 15. Sixth report DOMO 'Data Never Sleeps'. Available at: https://www. domo.com/learn/data-never-sleeps-6 